\title{
Non-uniform Signal Intensity Artifact
}

National Cancer Institute

\section{Source}

National Cancer Institute. Non-uniform Signal Intensity Artifact. NCI Thesaurus. Code C86996.

An artifact resulting from non-uniform signal intensities across the imaging field. 